\title{
Arterial hypertension in children
}

\author{
Maristella Santi ${ }^{\mathrm{a}, \text { Barbara Goeggel Simonetti }}{ }^{\mathrm{a}, \mathrm{b}}$, Corinna F.P. Leoni-Foglia ${ }^{\mathrm{a}}$, \\ Mario G. Bianchetti ${ }^{\mathrm{a}}$, and Giacomo D. Simonetti ${ }^{\mathrm{a}, \mathrm{c}}$
}

\begin{abstract}
Purpose of review
Although arterial hypertension is less common in children than in adults, there is growing concern about elevated blood pressure (BP) in children and adolescents not only because of the association of elevated values with the overweight epidemic, but also as cardiovascular functions are determined in childhood and track into adulthood. The purpose of the review is to discuss new aspects of childhood hypertension.

\section{Recent findings}

Guidelines advocate determining BP in children as part of routine health maintenance. This recommendation was recently subject to review by the US Preventive Services Task Force. It was concluded that evidence is insufficient to assess the benefits of this screening. In our opinion, however, assessing BP is part of any thorough physical examination.

Sophisticated approaches demonstrate the role of sympathetic nervous system overdrive in the field of sympathetic cardiovascular modulation of childhood arterial hypertension.
\end{abstract}

\section{Summary}

Elevated BP in children is increasing in frequency and is now recognized as having relevant short-erm and long-term consequences. Although efforts to address the childhood overweight epidemic may eventually reduce the number of young patients with hypertension, improved therapies for childhood hypertension also offer the potential for preventing or ameliorating early cardiovascular disease.

\section{Keywords}

adolescent, cardiovascular disease, children, epidemiology, hypertension, overweight, sympathetic nervous system

\section{INTRODUCTION}

Arterial hypertension affects about $20 \%$ of adults and is a leading cause of morbidity and mortality [1]. Although hypertension is less common in children than in adults, there is growing concern about elevated blood pressure (BP) and hypertension in children and adolescents because of the association of elevated BP with the overweight epidemic [2,3]. Moreover, it has been hypothesized that cardiovascular functions are determined in childhood and track into adulthood. Children with high BP values have a greater risk to have high (or too high) BP levels in adulthood, as indicated by the fact that almost half of adults with arterial hypertension had high BP values in childhood [3-5]. Tracking of BP begins in early childhood, at about the age of 5 years [6]. Consequently, prevention of adult cardiovascular diseases should begin early in childhood by counseling healthy lifestyle and avoiding preventable cardiovascular risk factors $\left[3,7^{\mathbf{*}}\right]$. Screening for high BP at regular intervals in childhood is currently a matter of debate and will be further discussed in this review together with the definition of arterial hypertension, its association with sympathetic nervous system overactivation and its consequences.

\section{Measurement of blood pressure and screening for hypertension in childhood}

In childhood, the recommended method for BP assessment is auscultatory with Korotkoff 1 applied for SBP and Korotkoff 5 applied for DBP.

If an oscillometric device is used, the monitor needs to be validated; additionally, if hypertension

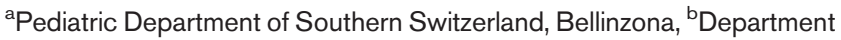
of Neurology and ${ }^{\mathrm{C}}$ Division of Pediatric Nephrology, Department of Pediatrics, Inselspital, Bern University Hospital, and University of Bern, Bern, Switzerland

Correspondence to Giacomo D. Simonetti, MD, Pediatric Department of Southern Switzerland, Ospedale Regionale Bellinzona e Valli, 6500 Bellinzona, Switzerland. Tel: +41 9181186 68; fax: +41 9181180 26; e-mail: giacomo.simonetti@eoc.ch
}

Curr Opin Cardiol 2015, 30:403-410

DOI:10.1097/HCO.0000000000000191 


\section{KEY POINTS}

- Guidelines advocate determining blood pressure in children during each healthcare episode. This recommendation was recently subject to review by the US Preventive Services Task Force, which concluded that evidence is insufficient to assess the benefits of screening in apparently healthy children and adolescents.

- There is a renewed interest in the field of sympathetic cardiovascular modulation of prehypertension, primary hypertension and chronic kidney disease.

- The syndrome in childhood hypertensive encephalopathy appears to be a form of posterior reversible encephalopathy syndrome, a reversible clinicoradiologic entity characterized by insidious onset of headache, confusion, visual disturbances and seizures associated with brain edema, which predominates in the posterior areas and in the white matter on imaging.

- Considering the high prevalence of secondary hypertension with a rather well-identified mechanism underlying high blood pressure in children and adolescents, a targeted approach to therapy is commonly advocated in an effort to maximize benefit.

is detected by the oscillometric method, it needs to be confirmed using the auscultatory method. Finally, the appropriate cuff size according to arm width (40\% of the arm circumference) and length $(4 \times 8 \mathrm{~cm}, 6 \times 12 \mathrm{~cm}, 9 \times 18 \mathrm{~cm}, 10 \times 24 \mathrm{~cm}$, to cover $80-100 \%$ of the individual's arm circumference) should be used [8]. Arterial hypertension in childhood is defined by at least three measurements at different times above the 95th centile adapted for age, height and sex. White coat hypertension and, more rarely, masked hypertension should be ruled out by 24-h ambulatory BP measurement (ABPM), particularly in older children. ABPM can nowadays be easily performed from the age of about 5 years onward and normal values for children are available [8].

In many industrialized countries, current guidelines advocate determining BP in children after the age of 3 years during each healthcare visit to screen for arterial hypertension [8,9]. This rather logical and intuitive suggestion was recently subject to intense scientific review by the US Preventive Services Task Force. The conclusion provided in the report is that 'current evidence is insufficient to assess the balance of benefits and harms of screening for elevated BP in apparently healthy children and adolescents to prevent subsequent cardiovascular disease in childhood or adulthood' [10]. This conclusion, which is largely based on the lack of data from longitudinal, randomized trials to support this practice, has been widely disputed [11",12-14,15"].

In our opinion, this review fails to acknowledge that measurement of BP, a cheap and integral part of any careful physical examination, is too infrequently recorded in everyday pediatric practice. In addition, in many countries routine metabolic blood screenings (more invasive and expensive) for inherited disorders that are rarer than arterial hypertension are performed in newborn infants. Furthermore, the limitations of the analysis performed by the US Preventive Services Task Force deserve some comment. In the following, we aim at discussing these three issues.

Physicians are trained to monitor the development and the growth of children and youth using, among others, weight, height, head circumference, body temperature, respiratory rate, pulse rate and BP. Pediatric reference tables for these parameters, including BP, are available worldwide. Meanwhile, pediatricians can avoid unfriendly BP tables and interpret user-friendly BP measurements by using a mnemonic formula, online calculators or applications for smartphones $[16,17]$.

BP measurement in children is part of routine health maintenance and is associated with no harm. In children with conditions increasing the risk of high BP - including premature birth, low birth weight, congenital heart disease, kidney diseases and treatment with drugs known to raise BP checks begin during infancy (Table 1). Further factors predisposing to arterial hypertension in schoolage children and adolescents are excessive body weight and a family history of elevated BP. In everyday clinical practice, BP is infrequently recorded in children and adolescents and, in our opinion, the conclusions raised by the US Preventive Services Task Force indirectly suggest that determination of BP is often unnecessary in children and adolescents. Our experience at a tertiary referral center with a catchment area of, approximately, one million people is in contrast to this conclusion. At this center, two or more asymptomatic cases of severe secondary hypertension with relevant target organ damage are encountered every year, supporting the notion that secondary arterial hypertension may go unrecognized in childhood [18].

Nowadays, most countries provide an expanded but rather expensive universal newborn metabolic screening for conditions such as adrenal hyperplasia, cystic fibrosis, galactosemia, hypothyroidism and phenylketonuria [19]. The frequency of arterial hypertension in children and adolescents is considered to be one in 50-100 individuals $[8,9]$. Thus, it appears surprising that rather expensive screening recommendations are used to identify rare or very 
Table 1. Conditions under which children 3 years of age or less should have blood pressure measured

Recurrent urinary tract infections
Hematuria or proteinuria
Known renal disease or urologic malformations
Family history of congenital renal disease
History of prematurity, very low birth weight, neonatal compli-
cations requiring intensive care
Congenital heart disease (repaired or not)
Malignancy
Solid organ or bone marrow transplant
Systemic diseases possibly associated with arterial hypertension
(e.g., neurofibromatosis, tuberous sclerosis, Marfan syndrome,
William-Beuren syndrome)
Management with drugs known to raise blood pressure

rare inherited diseases and that measuring $\mathrm{BP}$ at regular intervals is not advised for a quite common condition such as arterial hypertension, which is a substantial cause of heart hypertrophy, arterial stiffening and further organ damage that might be prevented by nonpharmacological and pharmacological intervention [20-23].

Finally, only 35 articles (out of 1000 potentially relevant articles) were included in the final analysis. As noted by two commentaries $\left[13,15^{*}\right]$, the Task Force recommendation fails to consider a considerable body of literature that includes relevant observational studies, specifically those addressing intermediate outcomes associated with childhood arterial hypertension, such as carotid artery thickening, left ventricular hypertrophy and urinary protein excretion [20-23].

We believe that the recommendation of the Task Force is inconclusive and flawed because the evidence was too narrowly selected, excluding short-term and relevant observational studies. Hence, we still advise routine screening for high BP at all well child visits, and hope that data from large pediatric cohorts with a long-term follow-up will support our impression.

\section{Sympathetic nervous system abnormalities in children and adolescents with tendency toward essential hypertension and kidney disease}

The sympathetic arm of the autonomic nervous system, which is mainly implicated in the homeostasis of involuntary processes in response to stressful or emergency situations, plays a critical role in the regulation of $\mathrm{BP}$ [24]. Its importance in the short-term regulation of arterial pressure via baroreflex mechanisms is well recognized. However, the role of the sympathetic nervous system in longerterm (days, months and years) BP modulation has been a focus of consideration since the 1970s [24]. Various studies have established that young participants with mild hypertension or normotensive offspring of hypertensive families often exhibit high heart rate and elevated cardiac output. Furthermore, several sympathetic-mediated abnormalities have been reported in these participants, including: a disturbed BP regulation after standing; an altered baroreceptor function; an exaggerated sensitivity of the forearm vasculature to exogenous norepinephrine or of the renal vessels to psychological stimuli; an increased responsiveness of BP to mental stress or dietary potassium restriction; and high circulating catecholamine concentrations during stress [24]. Approximately 30 years ago, a coauthor extensively investigated the BP responses to a stepwise increase in plasma norepinephrine or angiotensin II concentrations induced by exogenous infusions of the vasopressors in young adult patients with mild primary hypertension (and a normal BMI) and in normotensive participants with a negative or positive family history of essential hypertension. Patients and participants with positive history differed from those with negative history of hypertension by a decreased pressor dose of infused norepinephrine and by a different relationship between norepinephrine-induced changes in BP and concomitant plasma norepinephrine levels. In contrast, the pressor dose of infused angiotensin II and the relation between stepwise increasing plasma angiotensin II levels and induced changes in BP was similar in hypertensive patients and in the two groups of normotensive participants (Fig. 1). The aforementioned data indicate the existence of a selectively disturbed noradrenergic BP control in patients with mild primary hypertension and in normotensive offspring of hypertensive families $[25,26]$.

Since then, sophisticated biochemical (norepinephrine spillover), neurophysiological (direct recording of muscle sympathetic nerve traffic) and bioengineering (power spectral analysis of the heart rate signal) approaches have been developed to assess human sympathetic function, allowing new insights in this field $[24,27,28]$. These data demonstrate a renewed interest of investigators in the field of sympathetic cardiovascular modulation of arterial hypertension and chronic kidney disease. Currently available results indicate the following: sympathetic nervous system overdrive is often modulated by a genetic background; sympathetic overdrive appears very early in the course of primary hypertension; the degree of the sympathetic overactivation is directly related to the severity of 


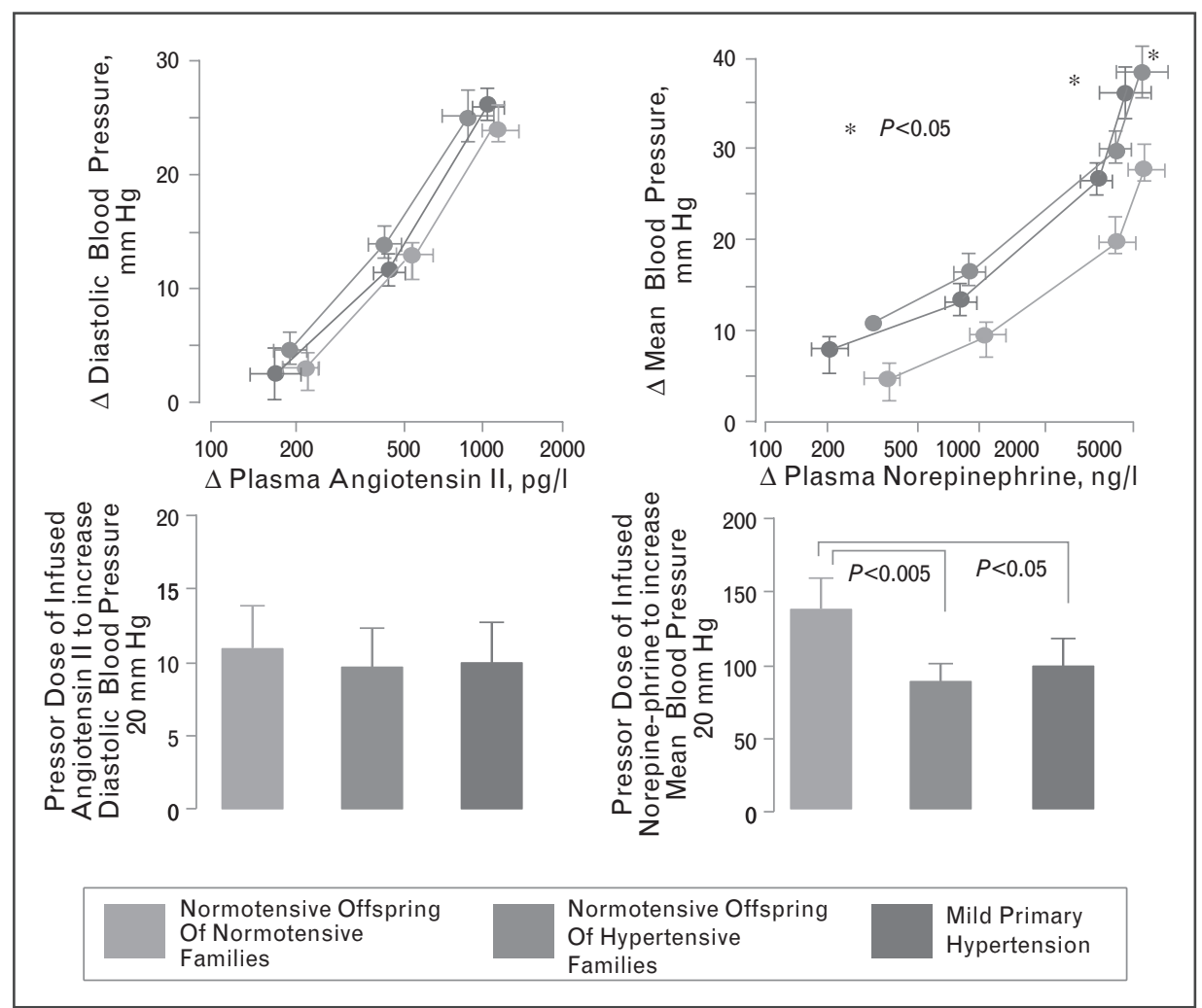

FIGURE 1. Blood pressure responses to a stepwise increase in plasma norepinephrine or angiotensin $\|$ concentrations induced by exogenous infusions of the vasopressors in 25 young adult patients (black) with mild primary hypertension (and a normal $B M I)$ and in normotensive participants with a negative $(N=25$; light gray) or with a positive $(N=25$; dark gray) family history of essential hypertension. Patients and participants with positive history differed from those with negative history of hypertension by a decreased pressor dose of infused norepinephrine (right lower panel) and by a different relationship between norepinephrine-induced changes in blood pressure and concomitant plasma norepinephrine levels (right upper panel). In contrast, the pressor dose of infused angiotensin II (left lower panel) and the relation between stepwise increasing plasma angiotensin II levels and induced changes in blood pressure (left upper panel) were similar in hypertensive patients and in the two groups of normotensive participants. The bars denote SEM.

primary hypertension; adrenergic activation displays an adverse impact on end-organ damage and cardiovascular morbidity; in chronic kidney disease, sympathetic activation occurs both in the earlier and in the more advanced clinical stages of the disease and is directly correlated to the severity of the disease; finally, overweight and weight gain are associated with increased sympathetic overdrive.

Catheter-based radiofrequency ablation of renal sympathetic nerves, also known as renal denervation, is a new, apparently promising, strategy that may substantially lower BP in patients with drugresistant hypertension. Regrettably, the utility of renal denervation has not been proven in drugresistant arterial hypertension [29"'].

\section{Short-term and long-term consequences of arterial hypertension in childhood}

Cardiovascular diseases such as stroke or myocardial infarction occur after several years of arterial hypertension. Consequently, these cardiovascular complications first appear in adulthood, indicating that is not possible to directly link BP levels in childhood with cardiovascular disease risk in adulthood. Nevertheless, a correlation between BP and surrogate markers of target-organ damage, such as left ventricular hypertrophy, arterial stiffness or increased urinary protein excretion, is demonstrable in children with arterial hypertension [3].

Severe hypertension in childhood mainly results from secondary causes, such as cardiac or renal disease. Acute symptoms or organ failure are normally not present in children with primary hypertension because in this age group primary hypertension is rather mild. Primary hypertension, as in adults, is mainly asymptomatic, or shows few symptoms such as headaches, shortness of breath, epistaxis, and changes in behavior or school performance.

Posterior reversible encephalopathy syndrome is a potentially reversible clinicoradiological entity characterized by insidious onset of headache, 
confusion, visual disturbances and seizures associated with brain edema, which predominates in the posterior white matter. Two major factors predispose to this entity in childhood: an acute and severe increase in BP and calcineurin inhibitors. Nephrotic syndrome and acute glomerulonephritides such as postinfectious glomerulonephritis, Immunoglobulin A ( $\operatorname{Ig} \mathrm{A})$ or purpura Schönlein-Henoch nephritis are the most common underlying conditions. As previously stated, altered consciousness (coma, stupor, lethargy and confusion), seizures, headache and visual disturbances are the most distinctive clinical presentation. Symptoms usually develop abruptly and resolve soon with proper management (BP reduction or discontinuation of the causative medication). Cranial MRI is the imaging modality of choice for detecting the entity [30]. The underlying pathophysiologic process appears to be a breakthrough of cerebral blood flow autoregulation, causing vasogenic edema.

The most significant long-term consequence of elevated BP in childhood is the ongoing hypertension into adulthood (tracking of BP [5]), therefore causing cardiovascular, cerebrovascular and renal disease later in life $[3,5,6,21,22]$. The most evident target-organ damage during childhood and adolescence is left ventricular hypertrophy $[8,9]$. About $50 \%$ of children and adolescents with arterial hypertension have a left ventricular mass index (LVMI) more than the 90th centile and 14\% have a LVMI more than the 99th centile [31]. An association between LVMI and systolic ambulatory BP has also been demonstrated in children; this association is stronger than with casual BP measurement $[32,33]$. Children with white coat hypertension have increased LVMI, representing an intermediate state between hypertensive and normotensive children [34]. Furthermore, prehypertension (BP values between the 90th and 95th centiles) represents an intermediate risk for the development of left ventricular hypertrophy [35].

Hypertensive retinopathy may be found in children with arterial hypertension. Retinal changes with retinal artery narrowing can be observed in children as young as $6-8$ years of age.

Preclinical atherosclerosis is assessed by measuring carotid intima-media thickness (cIMT). Several reports document the dependent relationship between cIMT values and childhood BP [22]. Other factors contributing to increased cIMT are BMI and low-density lipoprotein cholesterol [22,23]. Postmortem analysis in the Bogalusa Heart Study revealed that childhood SBP was correlated with fatty streaks and fibrous plaques in young adults [36]. Parameters of arterial distensibility, left ventricular mass, BMI and sedentary lifestyle in childhood were precursors of adult hypertension and target organ damage $\left[37,38^{-"}, 39,40^{\prime \prime}\right.$. Again, these data confirm that cardiovascular risk factors have origins in childhood. Another surrogate marker of impaired vascular function and arterial wall changes (i.e., calcification or atherosclerosis) is arterial stiffness, which is increased in childhood hypertension [41]. Obesity and arterial hypertension have a detrimental effect on arterial stiffness [42].

Childhood hypertension can also impair cognitive function and cerebral vascular reactivity. Lande et al. $[43,44]$ reported an association between arterial hypertension and decreased performance on tests of neurocognitive function, which should be adjusted for potential confounding effects of sleep disordered breathing [45]. Additionally, Adams et al. [46] reported learning and attention problems among children with pediatric primary hypertension. Finally, microalbuminuria is a marker of early renal damage. Childhood hypertension seems to affect microalbuminuria in children [47].

\section{Treatment of arterial hypertension in childhood}

Over the last years, management of childhood high $\mathrm{BP}$ has been addressed more rigorously than in the past, resulting in clearer consensus recommendations pertaining to appropriate drug management. In children and adolescents with uncomplicated primary hypertension, the goal of therapy should be $\mathrm{BP}$ reduction to less than the 95th percentile (despite therapeutic lifestyle modification). In the setting of concurrent diseases, such as diabetes mellitus, or target end-organ damage, BP should be lowered to less than the 90th percentile. On the basis of evidence that more aggressive control may be especially beneficial in slowing renal functional decline in nephropathic paticipants, the European guidelines advocate for more rigorous BP control, with target values less than the 75th percentile in children with chronic kidney disease and less than the 50th percentile in children with both kidney disease and increased urinary protein excretion [8].

Obviously, participants with asymptomatic primary hypertension may be managed successfully with lifestyle interventions, including extensive counseling regarding the importance of appropriate dietary choices, regular exercise and weight loss. Unfortunately, success may be arduous to achieve with this approach alone and antihypertensive medication is sometimes required.

Considering the high prevalence of secondary hypertension with a rather well-identified mechanism underlying high BP in children and adolescents, a targeted approach to therapy is commonly advocated in an effort to maximize benefit. In patients 
with concomitant diseases a specific drug may be particularly beneficial. For example, $\beta$-adrenergic blockade is advocated in children with coarctation of the aorta in the period before repair, as these agents reduce the degree of acute postintervention hypertension. In cases with persistent hypertension after correction, $\beta$-blockade is usually continued, although treatment with blockers of the reninangiotensin-aldosterone system may also be well tolerated and effective in these patients. Furthermore, in children with diabetes mellitus or kidney disease, therapy with a blocker of the renin-angiotensin-aldosterone system is advised, especially in cases with pathological urinary protein excretion. In patients affected with a chronic parenchymal kidney disease, the most common indication for antihypertensive drug management in childhood, blocking the renin-angiotensin-aldosterone system is very often insufficient to attain goal BP. In these cases, a diuretic (or a calcium channel-blocker) is added (a $\beta$-blocker is added only if the BP goal is still not achieved by combining three drugs) [8]. Table 2 shows the most frequently used antihypertensive drugs in childhood.

Finally, it is also important to consider potential adverse effects prior to initiating therapy. For example, noncardioselective $\beta$-adrenergic blockers are generally avoided in participants affected with reactive airway disease due to an increased risk of bronchospasm. On the other hand, there are significant fetal risks associated with the use of blockers of the renin-angiotensin-aldosterone during pregnancy (first trimester exposure might increase the risk of cardiovascular and central nervous system

Table 2. Antihypertensive drugs that may be administered once a day in the outpatient management of arterial hypertension in pediatric patients with chronic kidney disease

\begin{tabular}{lll} 
& & \multicolumn{2}{c}{ Daily dose $(\mathrm{mg})$} \\
\cline { 3 - 3 } Class & Agent & \multicolumn{2}{c}{ Body weight $(\mathrm{kg})$} \\
\cline { 3 - 3 } & $10-25$ & $25-40$
\end{tabular}

Thiazide diuretics

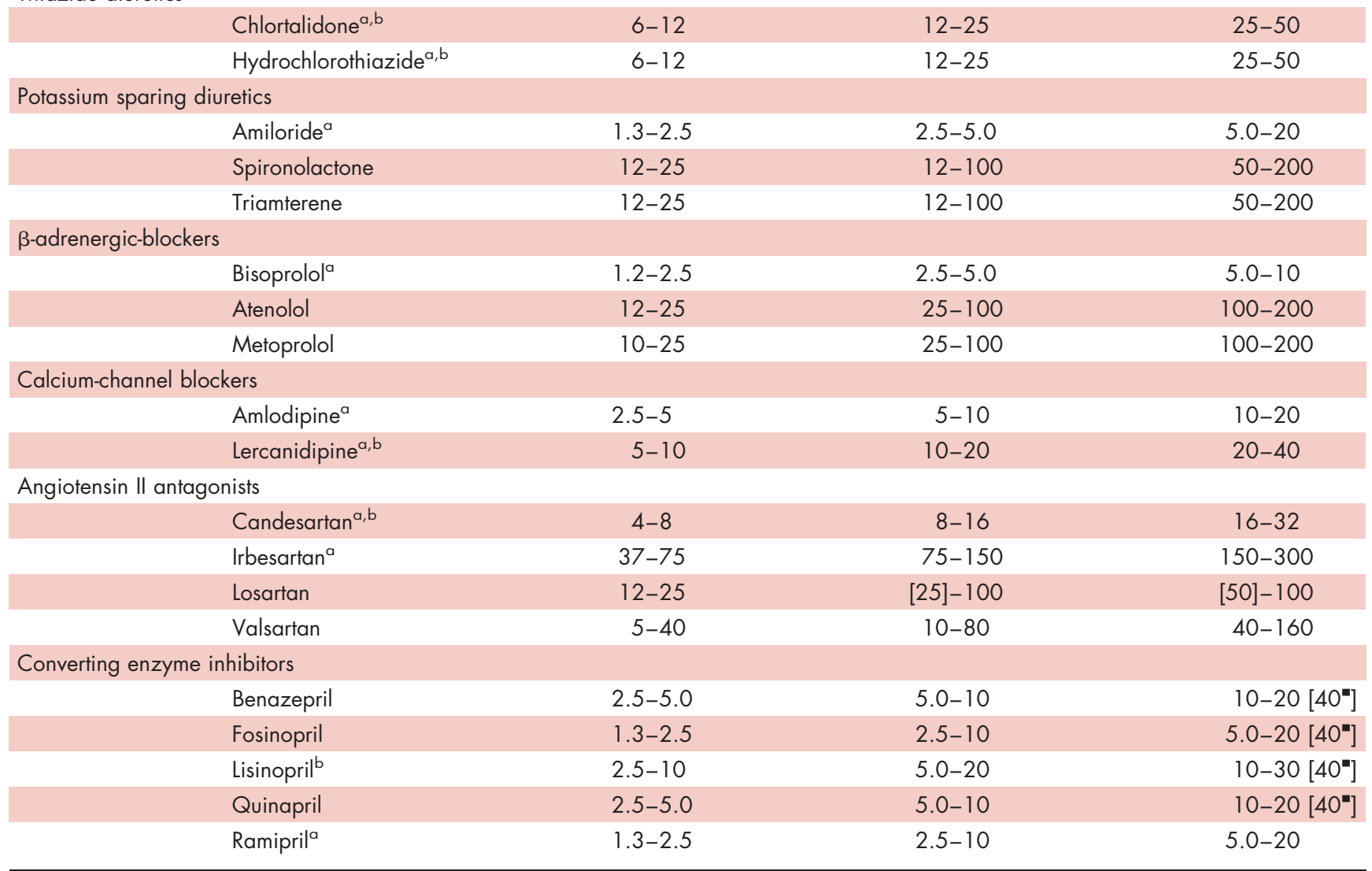

aThese drugs are very popular at our institutions.

bFavorable palatability. The prescription of drugs with sustained-release technologies that can reduce dosing frequency to once a day is not advised. The 'starting dose' is progressively escalated up to the 'maximum dose' every 3-6 weeks. 
malformations; second and third trimester exposure interferes with fetal renal function, decreasing fetal urine production and resulting in the fetal reninangiotensin system blockade syndrome, which is characterized by oligohydramnios, fetal pulmonary hypoplasia and deformational abnormalities) [48]. Consequently, discontinuation of these drugs is advised in women who present in early pregnancy, with substitution of an alternate agent if necessary. Furthermore, avoidance of drugs that block the renin-angiotensin-aldosterone system is suggested in women of childbearing age, for whom there are other antihypertensive drugs that would be well tolerated by the fetus.

\section{CONCLUSION}

Adult hypertension often begins in childhood. However, overt hypertension is noticeably different in childhood than it is in adults, and requires evaluation and management reflecting differences in the underlying pathophysiology. Although manifest cardiovascular disease related to hypertension is rare during childhood, early onset of this condition undoubtedly contributes to an acceleration of cardiovascular disease and target-organ damage, especially in the presence of the clustering of risk factors associated with overweight. We still recommend that children over 3 years of age should have their BP measured as part of routine healthcare.

\section{Acknowledgements}

None.

\section{Financial support and sponsorship}

None.

\section{Conflicts of interest}

There are no conflicts of interest.

\section{REFERENCES AND RECOMMENDED \\ READING}

Papers of particular interest, published within the annual period of review, have been highlighted as:

- of special interest

-1. of outstanding interest

1. Rosendorff C, Black HR, Cannon CP, et al. Treatment of hypertension in the prevention and management of ischemic heart disease: a scientific statement from the American Heart Association Council for High Blood Pressure Research and the Councils on Clinical Cardiology and Epidemiology and Prevention. Circulation 2007; 115:2761-2788.

2. McNiece $\mathrm{KL}$, Poffenbarger TS, Turner JL, et al. Prevalence of hypertension and prehypertension among adolescents. J Pediatr 2007; 150:640-644.

3. Bucher BS, Ferrarini $A$, Weber N, et al. Primary hypertension in childhood. Curr Hypertens Rep 2013; 15:444-452.

4. Bao W, Threefoot SA, Srinivasan SR, Berenson GS. Essential hypertension predicted by tracking of elevated blood pressure from childhood to adulthood: the Bogalusa Heart Study. Am J Hypertens 1995; 8:657-665.

5. Beckett LA, Rosner B, Roche AF, Guo S. Serial changes in blood pressure from adolescence into adulthood. Am J Epidemiol 1992; 135:1166-1177.
6. Chen $X$, Wang Y. Tracking of blood pressure from childhood to adulthood: a systematic review and meta-regression analysis. Circulation 2008; 117: 3171-3180.

7. Lava SA, Bianchetti MG, Simonetti GD. Salt intake in children and its con-

- sequences on blood pressure. Pediatr Nephrol 2014. [Epub ahead of print]

A review dealing with the relationship of salt intake and BP in children.

8. Lurbe E, Cifkova R, Cruickshank JK, et al. European Society of Hypertension. Management of high blood pressure in children and adolescents: recommendations of the European Society of Hypertension. J Hypertens 2009; 27:1719-1742.

9. National High Blood Pressure Education Program Working Group on High Blood Pressure in Children and Adolescents. The fourth report on the diagnosis, evaluation, and treatment of high blood pressure in children and adolescents. Pediatrics 2004; 114:555-576.

10. Moyer VA. U. S. Preventive Services Task Force. Screening for primary hypertension in children and adolescents: U. S. Preventive Services Task Force recommendation statement. Ann Intern Med 2013; 159:613619.

11. Brady TM, Redwine KM, Flynn JT. American Society of Pediatric Nephrology.

- Screening blood pressure measurement in children: are we saving lives? Pediatr Nephrol 2014; 29:947-950.

An article debating the controversy about BP screening in children.

12. Daniels SR, Gidding SS. Blood pressure screening in children and adolescents: is the glass half empty or more than half full? JAMA Pediatr 2013; 167:302-304.

13. Falkner B, Flynn J. Response to 'U.S. Preventive Services Task Force recommendation and pediatric hypertension screening: dereliction of duty or call to arms?'. J Clin Hypertens (Greenwich) 2014; 16:344-345.

14. Lo T, Malaga-Dieguez L, Trachtman HUS. Preventive Services Task Force recommendation and pediatric hypertension screening: dereliction of duty or call to arms? J Clin Hypertens (Greenwich) 2014; 16:342-343.

15. Urbina EM, de Ferranti $S$, Steinberger J. Observational studies may be more

- important than randomized clinical trials: weaknesses in US Preventive Services Task Force recommendation on blood pressure screening in youth. Hypertension 2014; 63:638-640.

A further article debating the controversy about BP screening in children.

16. Chiolero A, Paradis G. User-friendly tools to identify elevated blood pressure in children. Paediatr Child Health 2013; 18:63-64.

17. Simonetti GD, Rizzi M, Bianchetti MG. Simple references for managing arterial hypertension in children with kidney disease. J Hypertens 2010; 28:1109.

18. Hansen ML, Gunn PW, Kaelber DC. Underdiagnosis of hypertension in children and adolescents. JAMA 2007; 298:874-879.

19. Hoffmann GF, Lindner M, Loeber JG. 50 years of newborn screening. J Inherit Metab Dis 2014; 37:163-164.

20. Lai CC, Sun D, Cen R, et al. Impact of long-term burden of excessive adiposity and elevated blood pressure from childhood on adulthood left ventricular remodeling patterns: the Bogalusa heart study. J Am Coll Cardiol 2014; 64:1580-1587.

21. Hartiala $O$, Magnussen $C G$, Kajander $S$, et al. Adolescence risk factors are predictive of coronary artery calcification at middle age: the cardiovascular risk in young Finns study. J Am Coll Cardiol 2012; 60:1364-1370.

22. Juonala $M$, Magnussen $C G$, Venn $A$, et al. Influence of age on associations between childhood risk factors and carotid intima-media thickness in adulthood: the Cardiovascular Risk in Young Finns Study, the Childhood Determinants of Adult Health Study, the Bogalusa Heart Study, and the Muscatine Study for the International Childhood Cardiovascular Cohort (i3C) Consortium. Circulation 2010; 122:2514-2520.

23. Litwin M, Niemirska A, Sladowska-Kozlowska J, et al. Regression of target organ damage in children and adolescents with primary hypertension. Pediatr Nephrol 2010; 25:2489-2499.

24. Esler M. The sympathetic system and hypertension. Am J Hypertens 2000; 13:99S-105S.

25. Bianchetti MG, Beretta-Piccoli $C$, Weidmann P, Ferrier C. Blood pressure control in normotensive members of hypertensive families. Kidney Int 1986; 29:882-888.

26. Beretta-Piccoli C, Bianchetti MG, Pusterla C, Weidmann P. Cardiovascular hypersensitivity to norepinephrine in normotensive members of hypertensive families: influence of dietary sodium and potassium intake. Bibl Cardiol 1987; 42:144-151.

27. Joyner MJ, Charkoudian N, Wallin BG. Sympathetic nervous system and blood pressure in humans: individualized patterns of regulation and their implications. Hypertension 2010; 56:10-16.

28. DiBona GF. Sympathetic nervous system and hypertension. Hypertension 2013; 61:556-560.

29. Messerli $\mathrm{FH}$, Bangalore S. Renal denervation for resistant hypertension?

n Engl J Med 2014; 370:1454-1457.

This concise editorial addresses the role of ablation of renal sympathetic nerves in patients with arterial hypertension and the possible causes underlying the poor effect of this technique in a recently published blinded, sham-controlled study.

30. Ishikura K, Hamasaki Y, Sakai T, et al. Posterior reversible encephalopathy syndrome in children with kidney diseases. Pediatr Nephrol 2012; 27:375384. 
31. Daniels SR, Loggie JM, Khoury P, Kimball TR. Left ventricular geometry and severe left ventricular hypertrophy in children and adolescents with essential hypertension. Circulation 1998; 97:1907-1911.

32. Kollias A, Dafni M, Poulidakis E, et al. Out-of-office blood pressure and target organ damage in children and adolescents: a systematic review and metaanalysis. J Hypertens 2014; 32:2315-2331.

33. Sharma AP, Mohammed J, Thomas B, et al. Nighttime blood pressure, systolic blood pressure variability, and left ventricular mass index in children with hypertension. Pediatr Nephrol 2013; 28:1275-1282.

34. Lande MB, Meagher CC, Fisher SG, et al. Left ventricular mass index in children with white coat hypertension. J Pediatr 2008; 153:50-54.

35. Redwine KM, Daniels SR. Prehypertension in adolescents: risk and progression. J Clin Hypertens (Greenwich) 2012; 14:360-364.

36. Berenson GS, Srinivasan SR, Bao W, et al. Association between multiple cardiovascular risk factors and atherosclerosis in children and young adults. The Bogalusa Heart Study. N Engl J Med 1998; 338:1650-1656.

37. Li S, Chen W, Srinivasan SR, Berenson GS. Childhood blood pressure as a predictor of arterial stiffness in young adults: the bogalusa heart study. Hypertension 2004; 43:541-546.

38. Mikola H, Pahkala K, Rönnemaa T, et al. Distensibility of the aorta and carotid

- artery and left ventricular mass from childhood to early adulthood. Hypertension 2015; 65:146-152.

This study highlights the age-related decrease in vascular distensibility from childhood to early adulthood and its related risk factors.

39. Petkeviciene J, Klumbiene J, Simonyte $\mathrm{S}$, et al. Physical, behavioural and genetic predictors of adult hypertension: the findings of the Kaunas Cardiovascular Risk Cohort study. PLoS One 2014; 9:e109974.
40. Lai CC, Sun D, Cen R, et al. Impact of long-term burden of excessive adiposity

- and elevated blood pressure from childhood on adulthood left ventricular remodeling patterns: the Bogalusa Heart Study. J Am Coll Cardiol 2014; $64: 1580-1587$.

The findings of this study indicate that the adverse influence of excessive adiposity and elevated BP level on left ventricular hypertrophy begins in childhood.

41. Simonetti GD, VON Vigier RO, Wuhl E, Mohaupt MG. Ambulatory arterial stiffness index is increased in hypertensive childhood disease. Pediatr Res 2008; 64:303-307.

42. Saner $\mathrm{C}$, Simonetti GD, Wühl E, et al. Increased ambulatory arterial stiffness index in obese children. Atherosclerosis 2015; 238:185-189.

43. Lande MB, Kupferman JC, Adams HR. Neurocognitive alterations in hypertensive children and adolescents. J Clin Hypertens (Greenwich) 2012; 14:353-359.

44. Lande MB, Kupferman JC. Cognitive function in hypertensive children. Curr Hypertens Rep 2015; 17:508.

45. Lande MB, Hooper SR, Batisky DL, et al. Sleep disordered breathing as measured by SRBD-PSQ and neurocognition in children with hypertension. Am J Hypertens 2015; 28:552-558.

46. Adams HR, Szilagyi PG, Gebhardt L, Lande MB. Learning and attention problems among children with pediatric primary hypertension. Pediatrics 2010; 126:e1425-e1429.

47. Seeman T, Pohl M, Palyzova D, John U. Microalbuminuria in children with primary and white-coat hypertension. Pediatr Nephrol 2012; 27:461-467.

48. Bullo M, Tschumi S, Bucher BS, et al. Pregnancy outcome following exposure to angiotensin-converting enzyme inhibitors or angiotensin receptor antagonists: a systematic review. Hypertension 2012; 60:444-450. 